\title{
The role of adherence in mediating the relationship between depression and health outcomes
}

\author{
Rena R. Wing , Suzanne Phelan, Deborah Tate \\ Department of Psychiatry and Human Behavior, Brown Medical School, Miriam Hospital, Providence, RI, USA
}

\begin{abstract}
Depression has been linked to poor health outcome in a number of studies; however, the mechanism underlying this relationship has received little attention. This paper explores the possibility that adherence mediates the relationship between depression and outcome. Principal findings regarding the relationship between depression, adherence, and outcome are reviewed. The data suggest that depression is related, at least moderately, to poorer adherence to a variety of treatment components. The relationship between adherence and outcome is more difficult to establish. In addition, current data, albeit limited, do not support the hypothesis that adherence mediates the relationship between depression and outcome. An alternative model in which adherence precedes and influences both mood state and health outcome is discussed. Finally, possible explanations for these relationships are explored and suggestions for future research provided.
\end{abstract}

Keywords: Behavior; Mood; Diabetes; Exercise; Weight loss

\section{Introduction}

Depression has been associated with poor health outcomes in a variety of medical conditions. For example, depression has been linked to higher glycosylated hemoglobin in both type 1 and type 2 diabetes mellitus [1]. An important issue is to determine the mechanism underlying this relationship. One possibility, considered here, is that depressed patients are less adherent to their treatment regimens, and this lack of adherence produces poorer health outcomes (Fig. 1).
Studies of the relationship between depression and adherence have used a variety of approaches to assess depression or depressive symptomatology (ranging from questionnaires, such as the Center for Epidemiologic Studies-Depression questionnaire or the Beck Depression Inventory, to clinical interviews). Likewise, different aspects of adherence (adherence to diet, exercise, glucose monitoring, medication adherence, attendance at treatment sessions), different criteria for defining adherence, and different approaches to measuring adherence have been used. While acknowledging that these methodological differences may affect results, and recognizing that adherence to one aspect of the regimen may be unrelated to adherence to other aspects [2], we have focussed this paper on some of the general and consistent findings regarding the relationship between depression, adherence, and outcome. We have also considered other possible interrelationships among these variables, using exercise and obesity as examples.

The term "adherence" is used in preference to the term "compliance" throughout the paper to recognize the importance of the partnership between patient and provider in all aspects of medical care.

\section{Does depression affect adherence?}

Many studies have examined the association between depression and treatment adherence. It is well recognized that adherence to pharmacologic regimens for depression is very poor, with only $40 \%$ of those receiving an antidepressant prescription completing the 9-month recommended treatment [3]. Adherence to medical regimens is also poor in depressed patients. A meta-analysis of this literature included 12 studies published from 1968 to 1998 that measured depression and adherence to a medical regimen recommended by a nonpsychiatrist physician [4]. Six of these studies involved patients with end-stage renal disease 


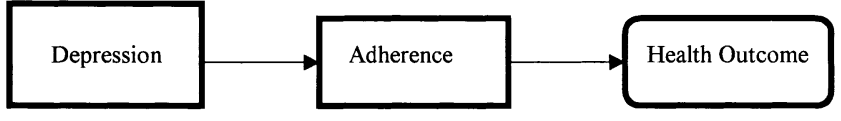

Fig. 1. Model showing adherence as the mediator of the relationship between depression and health outcome.

or renal transplants and six involved other medical diseases (angina, cancer, arthritis). The relationship between depression and nonadherence was found to be consistent across studies and of moderate size (Table 1). The odds that an individual would be nonadherent were three times greater for depressed patients than for nondepressed patients. In contrast, this meta-analysis found no consistent relationship between anxiety and treatment adherence.

Two recent studies reinforce this finding. In one study [5], 204 patients were interviewed for depression 3-5 days after a myocardial infarction and then re-contacted 4 months later to assess adherence to cardiac risk reduction recommendations. Patients who reported no current mood disorder were compared to those with major depression/dysthymia (based on the Structured Clinical Interview for Axis I Disorders). The depressed patients reported lower adherence to several aspects of the regimen, including exercise, low-fat diet, stress reduction, and prescription medication. No differences were observed for other aspects of the regimen such as stopping smoking, following a low sodium diet, or carrying medical supplies.

Another study [6] included 367 patients with diabetes who were seen in primary care clinics of a large health maintenance organization. Depressive symptoms were assessed with the Hopkins Symptom Checklist-90 Revised; adherence was assessed by self-report and by using an automated data collection system for refills of hypoglycemic medication. Higher levels of depressive symptoms were associated with poorer adherence to the diet (amount and type of food) and with more days of interruption of hypoglycemic medication therapy. There was no association between depressive symptom severity and self-reported adherence to exercise or glucose monitoring.

Thus, depression appears to be related, at least moderately, to poorer adherence to a variety of treatment components. It is now important to better understand why depressed patients are less adherent. There are a variety of possible explanations. Depressed patients report greater feelings of hopelessness (and thus may not expect treatment to be effective). They are often more socially isolated and social support has been related to adherence. Cognitive impairment in depressed patients may affect their memory of the health care prescriptions and recommendations given by the physician. Finally, depressed patients may not have the energy to carry out treatment recommendations.

It is also important to consider whether the strength of the association between depression and adherence is due, in part, to a methodological artifact. When asked to self-report their level of adherence, depressed patients may have poor self-perceptions and underestimate their actual level of adherence. Thus, it is particularly important to use objective measures of adherence (such as pill counts or pharmacy records as used in the Ciechanowski study [6]) in assessing the relationship between depression and adherence. Further research is needed to better understand why depressed patients are less adherent in order to develop effective approaches to enhance adherence.

\section{Does adherence affect outcome?}

The model described in Fig. 1 assumes that depression affects adherence and that adherence affects outcome. There is a large literature studying the effect of adherence on outcome, which will not be reviewed in detail here. In general, however, it appears that adherence to medication is related to outcome. A recent review [7] assessed the relationship between medication adherence and morbidity and mortality in patients who were at risk for coronary artery disease and congestive heart failure. Seven of 12 studies that compared hospitalization rates and mortality in adherent and nonadherent patients showed significant differences. Of particular note is the fact that adherence to placebo is also related to outcome.

It is more difficult to establish the relationship between adherence to behavioral recommendations and outcome. For example, in some weight loss studies, there are significant differences in weight loss between groups but no differences in dietary intake or exercise. This most likely reflects the difficulty in accurately measuring dietary intake and physical activity. Alternatively, the behaviors being studied may not be the behaviors that relate directly to outcome. For example, there may be little or no relationship between frequency of self-monitoring of blood glucose (SMBG) and glycosylated hemoglobin because the important behavior is not merely monitoring glucose levels but rather using the SMBG information to make adjustments in other aspects of the regimen.

\section{Does poor adherence mediate the relationship between depression and outcome?}

The question of whether adherence mediates the relationship between depression and outcome of diabetes,

Table 1

Meta-analysis of the relationship between depression and adherence

\begin{tabular}{lccl}
\hline Psychological variable & Number of studies & Unweighted & Effect size \\
\hline Depression (Total) & 12 & -0.27 & $P<.001$ \\
ESRD & 6 & -0.30 & $P=.008$ \\
Non-ESRD & 6 & -0.25 & $P=.005$ \\
Anxiety (Total) & 13 & -0.04 & $P=.59$ \\
\hline
\end{tabular}

Ref. [4]. 
obesity, or renal disease has received little empirical study. One approach to this question is to determine whether the association between depression and outcome is weakened or becomes nonsignificant after adjusting for level of adherence. Gary et al. [8] found that depressive symptomatology was associated with higher cholesterol, triglycerides, and glycosylated hemoglobin levels in Type 2 diabetic patients. Patients were asked to rate their adherence to various aspects of the diabetes regimen on fourpoint scales. Adjusting for level of adherence to diet, physical activity, smoking, glucose monitoring, and diabetes medication adherence did not reduce the effect of depression on these physiological outcome variables. Thus, this study provided no evidence that adherence mediated the relation between depression and outcome.

Another approach to determining whether adherence mediates the relationship between depression and outcome is to examine whether interventions that reduce depression and produce improvements in health outcome are associated with improvements in adherence. (If so, analyses adjusting for the improvement in adherence should reduce the association between changes in depression and changes in outcome.) Lustman et al. have conducted two intervention studies with depressed patients with diabetes; one study used an antidepressant medication (nortriptyline) to treat the depression [9] and the other used cognitive behavior therapy (CBT) [10]. In both studies, the intervention reduced depression and improved glucose control relative to the control condition. However, there was no evidence that reducing depression led to improved adherence, as measured by the use of home blood glucose monitoring. In fact, patients who participated in CBT showed worsening in their adherence to glucose monitoring relative to the control condition.

Thus, based on these few studies, there is no evidence to support the hypothesis that adherence mediates the relationship between depression and outcome. Further study with objective measures of adherence, clear criteria for defining adherence, and assessments of adherence to a variety of different behaviors is needed.

\section{An alternative model}

As discussed above, there are many studies showing a correlation between depressive symptomatology and behavior. These results are often interpreted as implying that depression causes poor adherence. It is important to recognize that in some situations, the direction of the relation may be reversed - the behaviors may precede and influence the mood state (Fig. 2). Hence by changing the behavior, it may be possible to change the mood state (rather than vice versa). Further, changing the behavior may influence the health outcome. There may also be indirect effects such that changing the behavior may influence health outcome through an effect on mood.

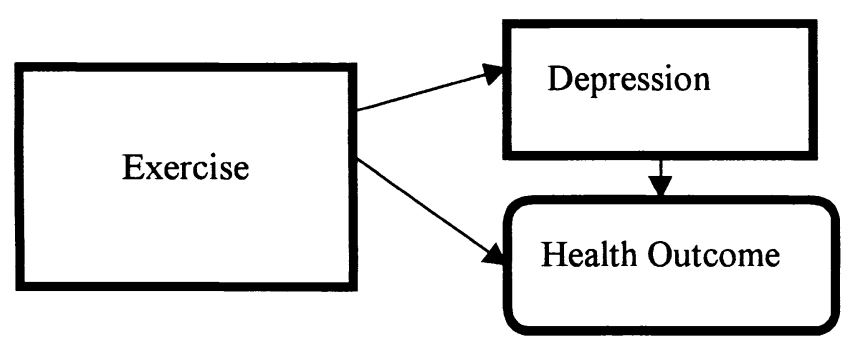

Fig. 2. Alternative model of the relationship between behavioral adherence, depression, and health outcome.

As an example consider exercise. A number of studies have shown an association between physical activity and depression [11]. Although depression may lead to more sedentary behavior, there are also several studies suggesting that low physical activity precedes, and is a risk factor for, subsequent depression. In the Harvard Alumni study, for example, physical activity level reported in 1962 and 1966 was associated with increased risk of physiciandiagnosed depression 23-27 years later [12]. Compared to those men who reported $<1000 \mathrm{kcal} /$ week of physical activity, those who reported $1000-2499 \mathrm{kcal} /$ week had a $17 \%$ reduction in risk of clinically recognized depression and those who were most active ( $>2500 \mathrm{kcal} /$ week) had a $28 \%$ reduction in risk.

Moreover, increasing exercise is an effective treatment for depression. In a recent study [13], 156 adults with major depressive disorder were randomly assigned to an exercise condition (three supervised exercise sessions per week for 16 weeks) or to depression medication (Sertraline), or to the combination of medication plus exercise. Remission rates after 4 months were comparable in the three conditions ( $60.4 \%$ of patients in the exercise group; $65.5 \%$ in the medication group; and $68.8 \%$ in the combined group had remission). Recently, 10-month follow-up data were presented for 133 of the original 156 patients [11]. Patients in the exercise group had significantly lower relapse rates than subjects in the medication group. In addition, exercising on one's own during follow-up was strongly associated (odds ratio $=0.49, P=.0009$ ) with reduced risk of a diagnosis of depression at the end of 10 months.

Obesity and weight control is also an interesting example. Depressive symptomatology, assessed at the start of a weight loss program, is not strongly or consistently associated with weight loss outcome, although in some studies, depression levels at baseline are associated with early termination of treatment. In contrast, participation in a behavioral weight loss program appears to produce marked, long-term improvements in depressive symptomatology. Patients report improvements in mood state within the first few weeks of a weight loss program, with improvements over the initial 6-month program apparently related to the magnitude of weight loss. However, interestingly, when participants are followed over time, improvement in mood persists even if the weight is regained. 
Foster et al. [14] studied 48 patients before and after a 6-month behavioral weight loss program and again at a 58-month follow-up. Body weight averaged 105.8, 84.7, and $109.4 \mathrm{~kg}$ at these three time points, respectively. Beck Depression Inventory scores were 12.7, 6.0, and 9.3 for the three time points, and the follow-up levels were significantly below $(P=.006)$ baseline. At follow-up, weight loss was unrelated to changes in mood. These data suggest that some aspect of the program (possibly the exercise component, the social support, or the cognitive behavioral approach), rather than weight loss per se, produced a positive change in mood state.

Changing physical activity level and/or weight can affect health outcomes directly and may also affect health outcomes via improvements in depression. There is extensive literature showing that physical activity level and body weight are related to risk of developing diabetes and/or cardiovascular disease and to mortality. Thus, changing these behaviors may have a far more dramatic effect on health outcomes than changing mood state.

\section{Conclusion and future research directions}

In conclusion, it is clear that there is a correlation between depressive symptomatology and adherence behaviors. It is time that the field moves beyond this simple fact and begins to ask why this correlation occurs and how it affects health outcomes. Below are some suggestions for future research.

\section{Better methods to assess adherence}

One of the major limitations in this field is the difficulty of setting criteria for defining adherence and objectively measuring this construct, especially adherence to complex behaviors such as diet. As noted above, selfreported behavior may be particularly problematic and potentially biased in depressed patients. Better ways to objectively assess key behaviors, such as diet and physical activity, are clearly needed. It is also important to establish criteria for determining when a patient is adherent, a task made more difficult by the fact that many behavioral prescriptions are given in general terms (e.g., "increase your physical activity"). It is also important to carefully specify the behaviors that we expect might be related to outcome. For example, rather than simply assessing frequency of self-monitoring of glucose, it is important to assess the use of SMBG information to regulate other aspects of the regimen.

\section{Research on whether adherence mediates the relationship between depression and outcome}

Future research should more specifically address the question of whether adherence mediates the relationship between depression and outcomes. Baron and Kenny [15] have proposed a methodological approach to studying mediators that would be helpful in this regard. Studies of the treatment of depression, where treatment improves health outcomes, should include objective measures of adherence. A variety of different aspects of adherence should be assessed (including adherence to both the depression and medical treatment regimens) since adherence to various components of the regimen are often not related to each other, and the combination of behaviors may be more strongly related to outcome than any one behavior [2]. Again, if the relationship between improvements in depression and health outcome is positive, researchers should determine whether this relationship is partially or fully explained by adjusting for adherence.

\section{Research to improve adherence in depressed patients}

Another approach to understanding the role of adherence in mediating the relationship between depression and outcome is to develop strategies to improve adherence to the medical treatment regimen for depressed patients and then determine whether the increased adherence improves health outcome. For example, simplifying the regimen or providing adherence reminders may be particularly helpful for patients with depression. Strategies that empower patients and encourage the development of an alliance between patients and providers may also improve adherence. In addition, examining whether improving adherence to pharmacologic or psychological treatment for depression has an effect on health outcome is an important area of inquiry.

4. Comparing interventions that are aimed at reducing depression with interventions that focus on behaviors such as exercise and weight control

Ultimately, the most important question is whether health outcomes are improved more dramatically by targeting depression or by targeting other behaviors that appear to be more directly related to the outcome (Fig. 2). Physical activity and weight loss have been shown to be related to the risk of developing diabetes and appear important in its treatment. Future research should determine whether diabetes health outcomes (e.g., glycosylated hemoglobin, lipids, blood pressure) are better addressed by reducing depression or by focussing on behaviors directly related to health outcome. Reducing depression with techniques such as CBT or medication may improve health outcome through several mechanisms, including, perhaps, increased physical activity and adherence to dietary recommendations. Alternatively, it may be more effective to directly focus on increasing physical activity or promoting weight loss; these behavior changes may simultaneously affect both depression and health outcome. 


\section{References}

[1] Lustman PJ, Anderson RJ, Freedland KE, DeGroot M, Clouse RE. Depression and poor glycemic control: a meta-analytic review of the literature. Diabetes Care 2000;23:934-41.

[2] Orme CM, Binik YM. Consistency of adherence across regimen demands. Health Psychol 1989;8:27-43.

[3] Depression Guideline Panel. Depression in primary care: detection and diagnosis, vol. 1. Rockville (MD): US Department of Health and Human Services, Public Health Service, Agency for Health Care Policy and Research, 1993 (AHCPR publication 93-0550).

[4] Dimatteo RM, Lepper HD, Croghan TW. Depression is a risk factor for noncompliance with medical treatment; meta-analysis of the effects of anxiety and depression on patient adherence. Arch Intern Med 2000;160:2101-7.

[5] Ziegelstein RC, Fauerbach JA, Stevens SS, Romanelli J, Richter DP, Bush DE. Patients with depression are less likely to follow recommendations to reduce cardiac risk during recovery from a myocardial infarction. Arch Intern Med 2000;160:1818-23.

[6] Ciechanowski PS, Katon WJ, Russo JO. Depression and diabetes: impact of depressive symptoms on adherence, function, and costs. Arch Intern Med 2000;3278-85.

[7] McDermott MM, Schmitt B, Wallner E. Impact of medication nonadherence on coronary heart disease outcomes. Arch Intern Med 1997;1921-9.

[8] Gary TL, Crum RM, Cooper-Patrick L, Ford D, Brancati FL. Depres- sive symptoms and metabolic control in African-Americans with type 2 diabetes. Diabetes Care 2000;23:23-9.

[9] Lustman PJ, Griffith LS, Clouse RE, Freedland KE, Eisen S, Rubin EH, Carney RM, McGill JB. Effects of nortriptyline on depression and glycemic control in diabetes: results of a double-blind, placebocontrolled trial. Psychosom Med 1997;59:241-50.

[10] Lustman PJ, Griffith LS, Freedland KE, Kissel SS, Clouse RE. Cognitive behavior therapy for depression in type 2 diabetes mellitus: a randomized, controlled trial. Ann Intern Med 1998;129:613-21.

[11] Babyak M, Blumenthal JA, Herman S, Khatri P, Doraiswamy M, Moore K, Craighead E, Bladewicz T, Krishnan R. Exercise treatment for major depression: maintenance of therapeutic benefit at 10 months. Psychosom Med 2000;62:633-8.

[12] Paffenbarger R, Leung R. Physical activity and personal characteristics associated with depression and suicide in American college men. Acta Psychiatr Scand 1994;377:16-22.

[13] Blumenthal JAB, Babyak M, Moore K, Craighead WE, Herman S, Khatri P, Waugh R, Napolitano MA, Forman LM, Appelbaum M, Doraiswamy PM, Krishnan KR. Effects of exercise training on older patients with major depression. Arch Intern Med 1999;159:2349-56.

[14] Foster GD, Wadden TA, Swain RMS, Stunkard AJ, Vogt RA. The Eating Inventory in obese women: clinical correlates and relationship to weight loss. Int J Obes 1998;22:785.

[15] Baron RK, Kenny DA. The moderator-mediator variable distinction in social psychological research: conceptual, strategic, and statistical considerations. J Pers Soc Psychol 1986;51:1173-82. 